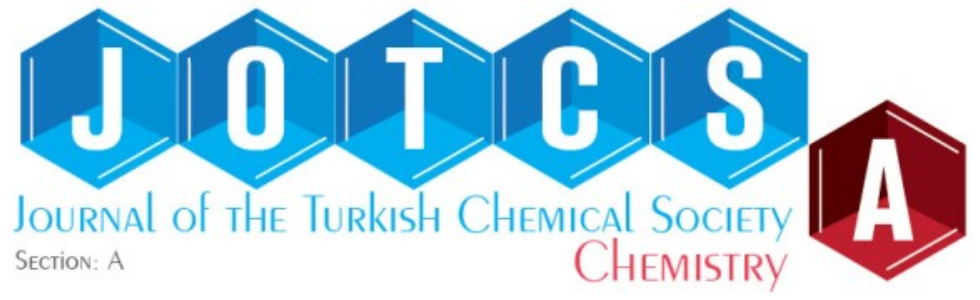

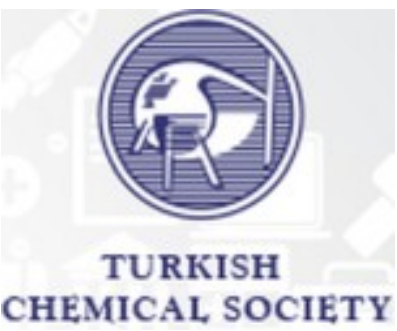

\title{
Drug Repurposing Against SARS-CoV-2: Targeting Nsp16-Nsp10 Interaction
}

\author{
Sefer Baday* $M$ iD
}

Applied Informatics Department, Informatics Institute, Istanbul Technical University, Ayazaga Campus, Maslak, 34469 Istanbul-Turkey

\begin{abstract}
Drug repurposing studies have played a crucial role in fighting the Covid-19 pandemic. Discovering a new drug molecule for disease takes a very long time. However, repurposing a drug molecule developed for another disease can accelerate new treatments for a disease. Thus, several drug repurposing studies were carried out targeting essential proteins for SARS-CoV-2. Nsp16-Nsp10 interaction was targeted in this work since this interaction is needed for SARS-CoV-2 to evade the human immune system. Therefore, docking calculations of approved 2126 drug molecules obtained from the Drugbank database were performed using the AutoDock Vina program. These docking calculations, drugs Ledipasvir Elbasvir, Venetoclax, Digitoxin, Irinotecan, Dexamethasone, Acetyldigitoxin, Dactinomycin, Lumacaftor, and Simeprevir, have the highest docking scores. Significant interactions for these drug molecules were presented.
\end{abstract}

Keywords: Covid-19, SARS-CoV-2, Docking, Drug Repurposing, Nsp16.

Submitted: April 20, 2021. Accepted: August 05, 2021.

Cite this: Baday S. Drug Repurposing Against SARS-CoV-2: Targeting Nsp16-Nsp10 Interaction. JOTCSA. $2021 ; 8(3): 933-40$.

DOI: https://doi.org/10.18596/jotcsa.923455.

*Corresponding author. E-mail: badays@itu.edu.tr.

\section{INTRODUCTION}

Since the outbreak of the Covid-19 pandemic, more than 130 million people have been infected by SARS-CoV-2 as of April 2021 (1). Even though several vaccines have been developed to combat SARS-CoV-2, existing mutations and prospective mutations necessitate alternative therapeutics (2). Since discovering a novel small molecule against a drug target could take a significant amount of time, drug repurposing studies were performed to speed up the process of finding a drug molecule against SARS-CoV-2 (3). In drug repurposing studies, alternative drug targets are needed due to two important reasons: The loss of efficacy resulting from mutations and enhancing efficacy due to combination therapies. Also, some molecules might not show efficacy in clinical trials despite having promising pre-clinical trial results. Thus, the diversity and abundance of available therapies for the treatment of covid-19 patients is important. Many attempts have been made to contribute the
Covid-19 arsenal. As an example, a detailed interaction study was performed and 332 important protein-protein interactions were reported to be potential drug targets (4). Among apparent targets such as spike Receptor Binding Domain (RBD), SARS-CoV-2 main protease, and transmembrane serine protease 2 (TMPRSS2), Non-structural protein 16 is a promising target for drug repurposing studies (5-7).

Non-structural protein 16 (Nsp16) methylates the viral RNA cap so that viral RNA cannot be distinguished from the host RNA. For the enzymatic activity, Nsp16 needs Non-structural protein 10 (Nsp10). Nsp16 and Nsp10 form a heterodimer complex structure (8). The Nsp16/Nsp10 complex transfers a methyl group from Sadenosylmethionine (SAM) cofactor to the $2^{\prime}$ hydroxyl of ribose sugar of viral mRNA. Interestingly human homolog of Nsp10, CMTr1 does not need a partner for the methylation activity. Since SAM cofactor is also needed in several human 
methyltransferases, targeting SAM binding site for the drug discovery might not be an ideal strategy due to potential off-target side effects (9). Drug molecules targeting SAM binding site of Nsp16 might also bind human CMTr1. An alternative strategy would be preventing the formation of the Nsp16/Nsp10 complex structure. Thus, in this work, drug repurposing is applied on SARS-CoV-2 Nsp16/Nsp10 interaction site.

Molecular docking calculations are one of the fundamental methods to predict the binding of a ligand to a protein (10-12). There have been several drug repurposing studies that target Nsp16 protein. Tazikeh-Lemeski et al. selected 1516 FDA-approved drug molecules based on similarity with SAM and docked these molecules into the SAM binding groove of Nsp16 (13). Quan Yang and co-workers screened 7,173 stereoisomers of 4,574 approved ligands obtained from the MTiOpenScreen database. They carried out docking of these molecules in the SAM binding pocket (14). Vijayan et al. performed docking of 2,100 FDA drugs from ZINC and 400 compounds from Spec database, and 1,600 drugs from DrugBank into the SAM binding site (15). In these drug repurposing studies, docking calculations were performed on the SAM binding site. However, targeting Nsp16-Nsp10 interaction has not been the focus yet. Therefore, in this work, we aim to predict potential drug molecules which might hamper the interaction of Nsp16 with Nsp10. To do so, the Nsp16-Nsp10 interface is selected as the center in the docking calculations.

\section{MATERIAL AND METHODS}

\section{Protein and Ligand preparation}

The structure of the Nsp16-Nsp10 complex was obtained from the protein data bank (PDB ID: $6 \mathrm{~W} 4 \mathrm{H})$. All crystallographic water molecules and non-amino acid molecules were removed from the structure. Protonation states of the amino acids of Nsp16 were determined using python implementation of PROPKA3 (16, 17). Hydrogen atoms were added using the AutoDockTools program (18). The docking box was centered on the Nsp16-Nsp10 interface (Figure 1). The center for the grid box was selected to be $76,25,16$ on $X, Y$ and $Z$ axis by manually orienting the center for the grid box. Note that these coordinates are based on the $6 \mathrm{~W} 4 \mathrm{H} . \mathrm{pdb}$ structure. The dimensions of the docking box were set to be 20,35 , and $25 \AA$ in the $X, Y$, and $Z$-axis, respectively. Docking calculations were performed using the AutoDock Vina program (19). The exhaustiveness parameter is set to 24 in these calculations. Information of the 2126 drug molecules was downloaded from the DrugBank in the SDF file format as of April 2021 (20). Threedimensional structures of the ligands were created and converted into the PDBQT file format using the Open Babel software (21). Ligand interaction diagrams were produced using Maestro (academic version) program (22).

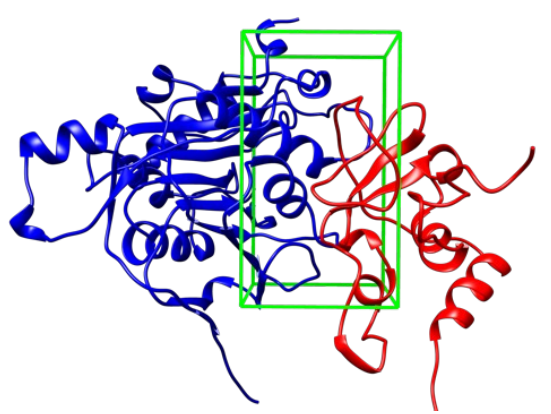

Figure 1: Visualization of grid box used in docking calculations. Blue: Nsp16, red: Nsp10, and Green: Docking box.

\section{RESULTS AND DISCUSSION}

Prior to screening of drug molecules, re-docking of SAM molecule was performed to validate the docking protocol. RMSD difference between the binding pose obtained by the docking calculation and the binding pose observed in the crystal structure was calculated as $0.647 \AA$ (Figure 2 ). The distribution of the docking scores for tested drug molecules is given in Figure 3. Docking scores were populated around $-6.1 \mathrm{kcal} / \mathrm{mol}$. Top-ranked compounds as a result of docking screening are summarized in Table 1. 


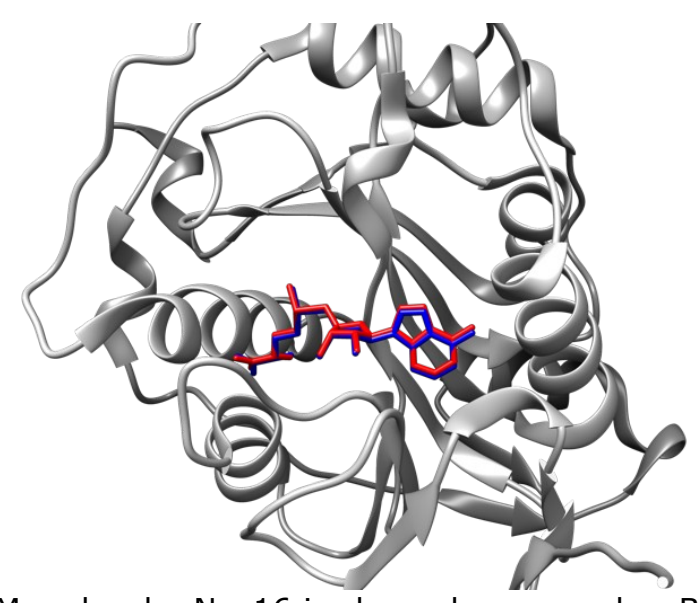

Figure 2: Re-docking of SAM molecule. Nsp16 is shown by gray color. Binding pose of SAM obtained by docking calculation is shown by red color. SAM conformation observed in the crystal structure is shown by blue color.

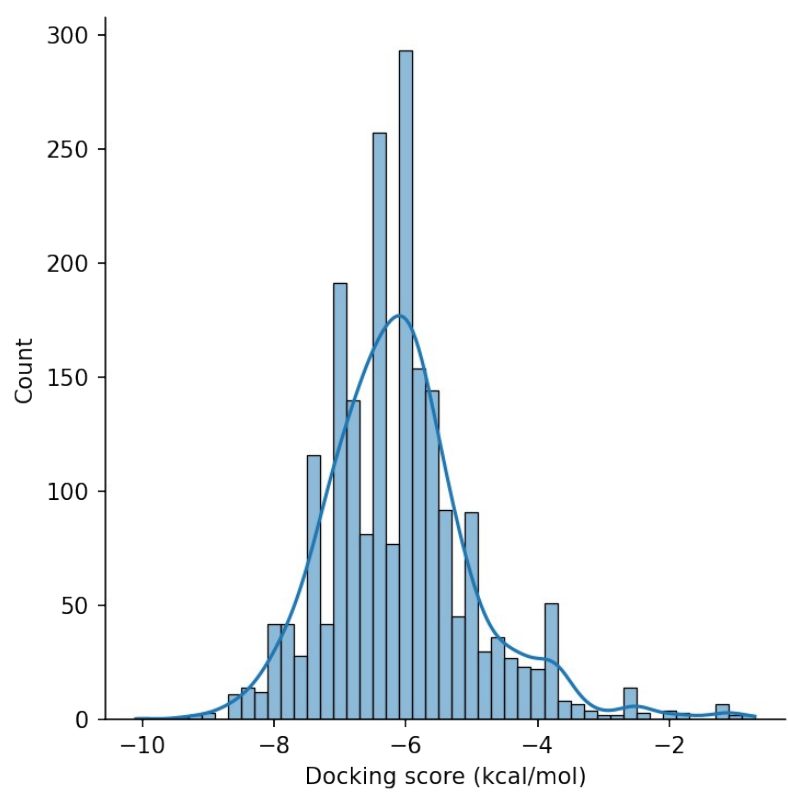

Figure 3: Distribution of docking scores.

Table 1: Docking scores for the top-ranked drug molecules.

\begin{tabular}{|c|c|c|c|}
\hline & Drug Name & $\begin{array}{l}\text { Docking Score } \\
\text { (kcal/mol) }\end{array}$ & Disease or Condition \\
\hline $\mathbf{1}$ & Ledipasvir & -10.1 & Hepatitis C \\
\hline 2 & Elbasvir & -9.4 & Hepatitis C \\
\hline 3 & Venetoclax & -9.2 & lymphocytic leukemia \\
\hline 4 & Digitoxin & -9.2 & $\begin{array}{l}\text { Congestive cardiac insufficiency, arrhythmias, and cardiac } \\
\text { failure }\end{array}$ \\
\hline 5 & Irinotecan & -9.0 & metastatic colorectal cancer \\
\hline 6 & Dexamethasone & -9.0 & $\begin{array}{l}\text { endocrine, rheumatic, collagen, dermatologic, allergic, } \\
\text { ophthalmic, gastrointestinal, respiratory, hematologic, } \\
\text { neoplastic, edematous }\end{array}$ \\
\hline 7 & Acetyldigitoxin & -8.9 & Congestive cardiac failure \\
\hline 8 & Dactinomycin & -8.7 & $\begin{array}{l}\text { Wilms' tumor, childhood rhabdomyosarcoma, Ewing's } \\
\text { sarcoma and metastatic, nonseminomatous testicular cancer }\end{array}$ \\
\hline 9 & Lumacaftor & -8.7 & cystic fibrosis (CF) \\
\hline 10 & Simeprevir & -8.7 & hepatitis C \\
\hline
\end{tabular}


In these docking calculations, the Ledipasvir molecule has the highest absolute docking score of $10.1 \mathrm{kcal} / \mathrm{mol}$. Ledipasvir is used as an antiviral for treating chronic hepatitis $\mathrm{C}$. Important interactions between Ledipasvir and Nsp16 are given in Figure 4. Ledipasvir forms hydrogen bond interactions with Gln6885 and Ser6903 and forms hydrophobic contacts with Val6842, Ala6843, Ala6881, Phe7043, and Leu7042. After Ledipasvir, Elbasvir has the second-highest docking score of $-9.4 \mathrm{kcal} / \mathrm{mol}$. Elbasvir is also an antiviral used to treat chronic hepatitis C. Elbasvir makes hydrogen bonds with Ser6903 and Asp6904 and makes hydrophobic contacts with Ala6881, Leu7042, Ala6905, and Pro6835. Venetoclax has the third-highest docking score of $-9.2 \mathrm{kcal} / \mathrm{mol}$. Venetoclax is a BCL-2 inhibitor and used for the treatment of chronic lymphocytic leukemia. Venetoclax forms hydrogen bonds with Arg6884 and has hydrophobic contacts with Pro6878, Ala6877, Val6876, and Leu6887. Digitoxin also has a docking score of $-9.2 \mathrm{kcal} / \mathrm{mol}$. Digitoxin is a cardiac glycoside used to treat atrial fibrillation, chronic cardiac failure, and arrhythmias. Digitoxin forms hydrophobic contacts with Nsp16 through Pro6888, Leu6887, Val6882, Ala6881, Val6882, Ile6838, Pro6835, Phe7043, and Leu7042 residues. Irinotecan has a docking score of -9.0 $\mathrm{kcal} / \mathrm{mol}$. Irinotecan is a topoisomerase-1 inhibitor used for the treatment of colorectal cancer. Irinotecan makes hydrogen bond interaction with Arg6884 and forms hydrophobic contacts with Pro6888, Leu6887, Ala 6877, Val6876, Val6842 and Val6902 residues. Dexamethasone also has -9.0 $\mathrm{kcal} / \mathrm{mol}$ predicted binding affinity. Dexamethasone is an anti-inflammatory and immunosuppressant molecule used to treat a variety of diseases and conditions such as arthritis, blood/hormone disorders, and allergic reactions. Dexamethasone has hydrophobic contacts with Val6882, Ala6881,
Val6842, Ile6838, and Pro6835. Acetyldigitoxin is an acetyl derivative of digitoxin and has a binding score of $-8.9 \mathrm{kcal} / \mathrm{mol}$. Acetyldigitoxin is used for the treatment of congestive cardiac failure. Acetyldigitoxin has hydrophobic interactions with Val6882, Ala6881, Val6842, Ile6838, Pro 6835, Phe7043, and Leu7042. Dactinomycin has -8.9 $\mathrm{kcal} / \mathrm{mol}$ predicted binding affinity. Dactinomycin is a chemotherapy agent used to treat various cancers such as Wilms' tumor, childhood rhabdomyosarcoma, and Ewing's sarcoma. Dactinomycin has hydrophobic contacts with Ala6881, Val6882, Val6842, Ile6838, and Val6876 amino acids of Nsp16. Lumacaftor also has a binding affinity of $-8.7 \mathrm{kcal} / \mathrm{mol}$. Lumacaftor is used for the treatment of cystic fibrosis. Lumacaftor has hydrophobic interaction with Gln6885 and hydrophobic contacts with Pro7049, Leu6887, Pro6888, and Met7045. Simeprevir has a docking score of $-8.7 \mathrm{kcal} / \mathrm{mol}$. Simeprevir is an antiviral used to treat hepatitis C. Simeprevir has hydrophobic interactions with Val6876, Ala6877, Pro6878, Ala6881, Leu6887, Ala6905, and Met6839 amino acids. When we compare these top-10 ranked compounds with the predicted compound due to other drug-repurposing studies by Tazikeh-Lemeski et al.; and Vijayan et al., our predictions do not overlap with predictions of these studies (15). This situation is normal since, in those drug repurposing studies against Nsp16, SAM binding site was selected for docking calculations. We also investigated how docked molecules overlap the Nsp16-Nsp10 interface. To do so, we visualized the Nsp16-Nsp10 complex with docked structures of a few drug molecules in Figure 6 . It is seen that docked molecules cover the Nsp16-Nsp10 interaction surface well. A summary of the amino acids forming important interactions with drug molecules is presented in Table 2 .

Table 2: Summary of important amino acids forming hydrophobic contacts and hydrogen bonds with drug molecules.

\begin{tabular}{|c|c|c|}
\hline Drug Name & Hydrophobic contacts & Hydrogen bonding \\
\hline Ledipasvir & Val6842, Ala6843, Ala6881, Phe7043 & Gln6885, Ser6903 \\
\hline Elbasvir & Ala6881, Leu7042, Ala6905, Pro6835 & Ser6903, Asp6904 \\
\hline Venetoclax & Pro6878, Ala6877, Val6876, Leu6887 & Arg6884 \\
\hline Digitoxin & $\begin{array}{l}\text { Pro6888, Leu6887, Val6882, Ala6881, Val6882, Ile6838, } \\
\text { Pro6835, Phe7043, Leu7042 }\end{array}$ & \\
\hline Irinotecan & Pro6888, Leu6887, Ala 6877, Val6876, Val6842, Val6902 & Arg6884 \\
\hline Dexamethasone & Val6882, Ala6881, Val6842, Ile6838, Pro6835 & \\
\hline Acetyldigitoxin & $\begin{array}{l}\text { Val6882, Ala6881, Val6842, Ile6838, Pro 6835, Phe7043, } \\
\text { Leu7042 }\end{array}$ & \\
\hline Dactinomycin & Ala6881, Val6882, Val6842, Ile6838, Val6876 & \\
\hline Lumacaftor & Pro7049, Leu6887, Pro6888, and Met704 & Gln6885 \\
\hline Simeprevir & $\begin{array}{l}\text { Val6876, Ala6877, Pro6878, Ala6881, Leu6887, Ala6905, } \\
\text { Met6839 }\end{array}$ & \\
\hline
\end{tabular}




\section{Ledipasvir}

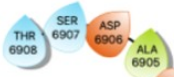

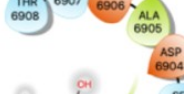

\section{Elbasvir}

Venetoclax
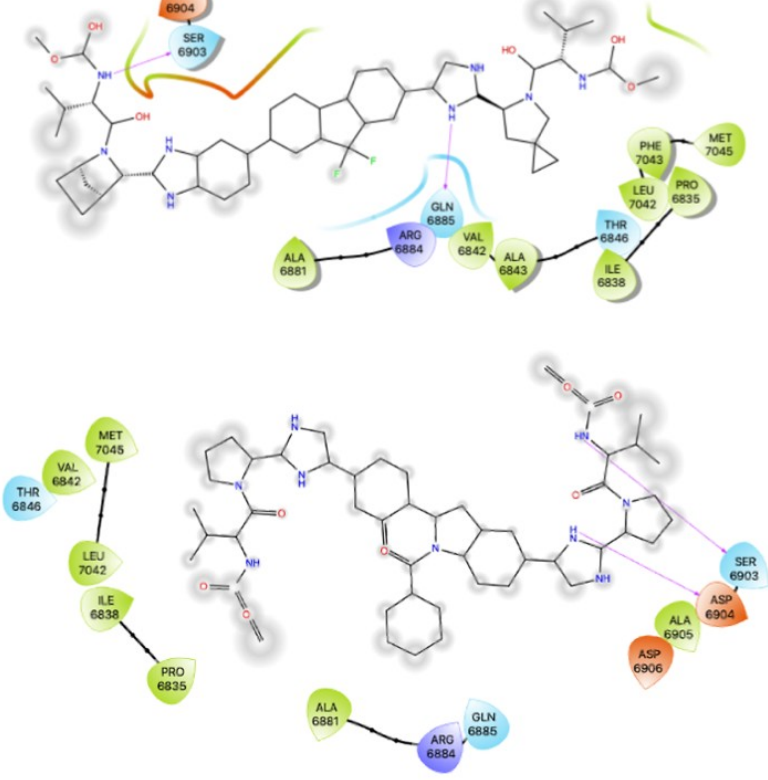

Digitoxin

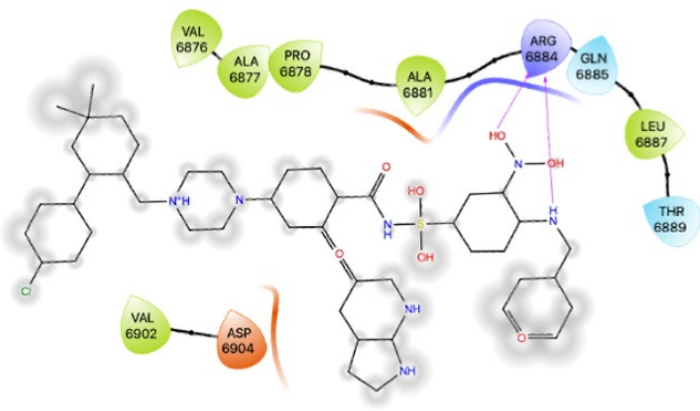

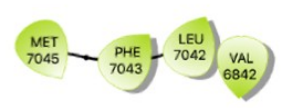
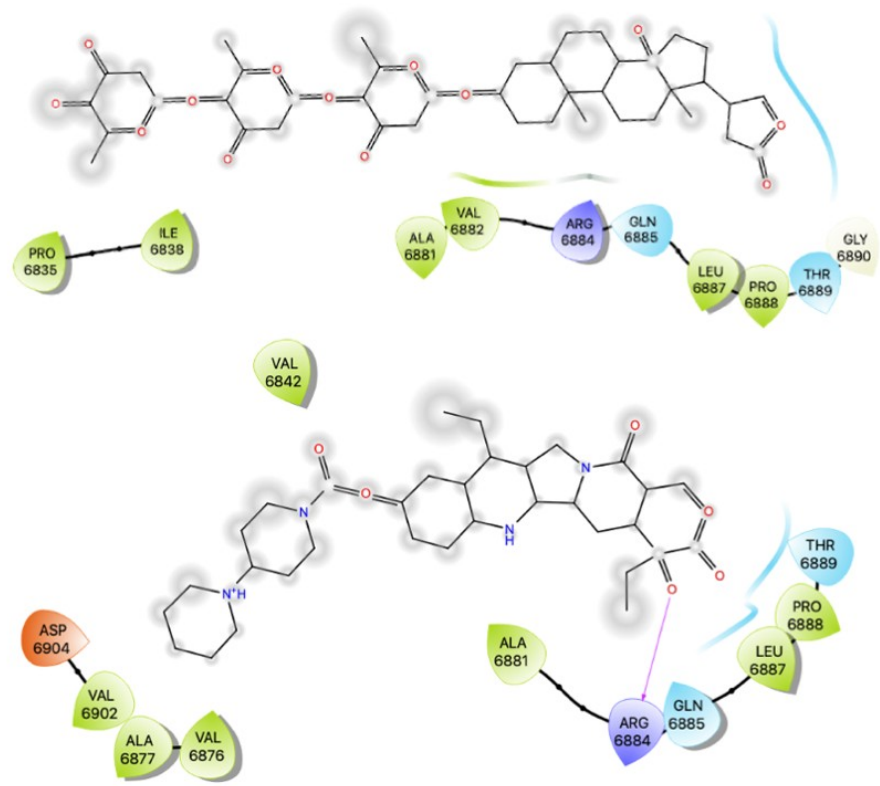

Figure 4: Ligand interaction diagrams using the docked structures of the drug molecules. 
$\underset{\substack{\text { THR } \\ 6846}}{(\mathrm{~s})}$

Dexamethasone

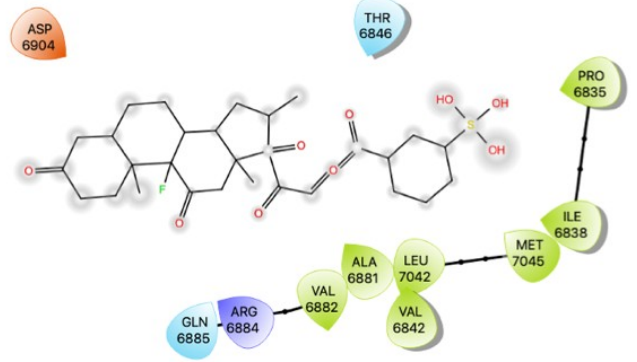

Acetyldigitoxin

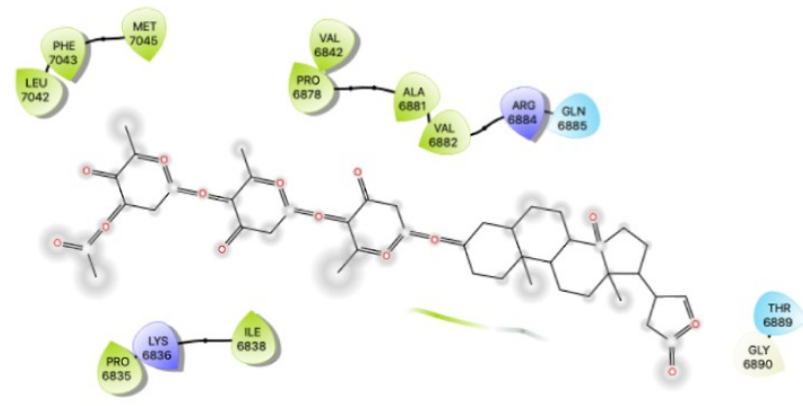

Dactinomycin

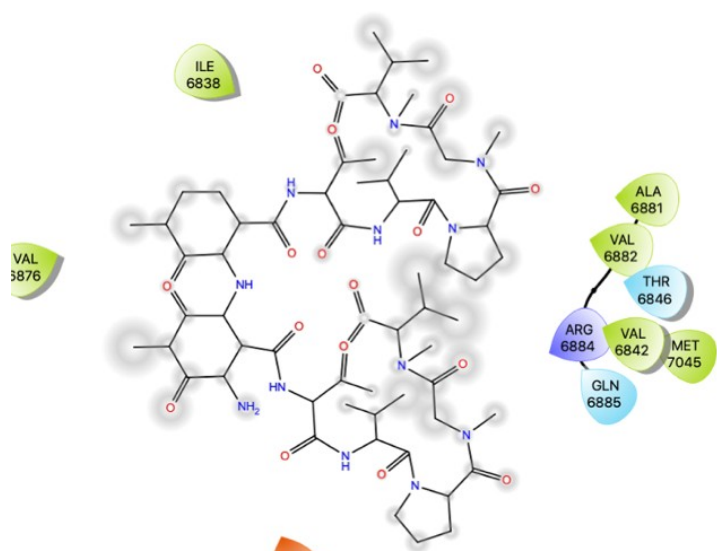

THR
6889

\section{Lumacaftor}
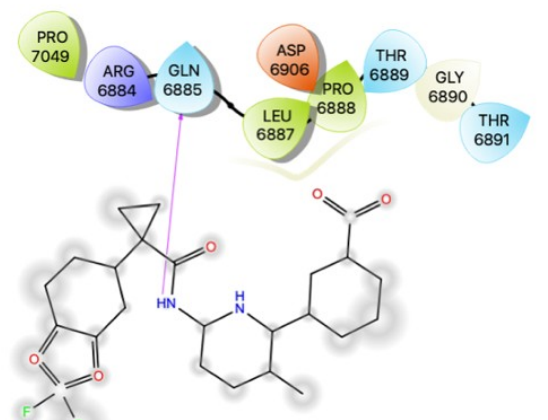

MET
7045

Simeprevir

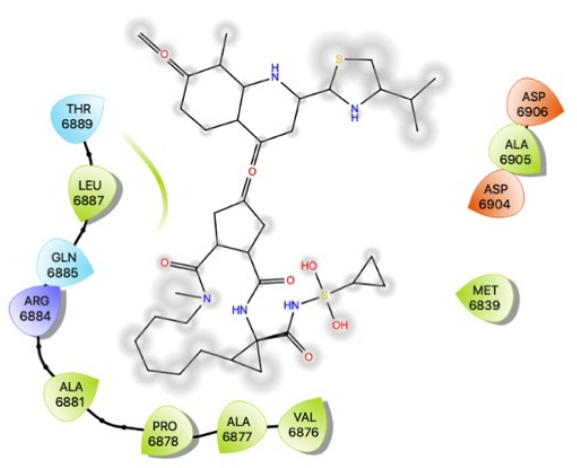

Figure 5: Ligand interaction diagrams using the docked structures of the drug molecules. 

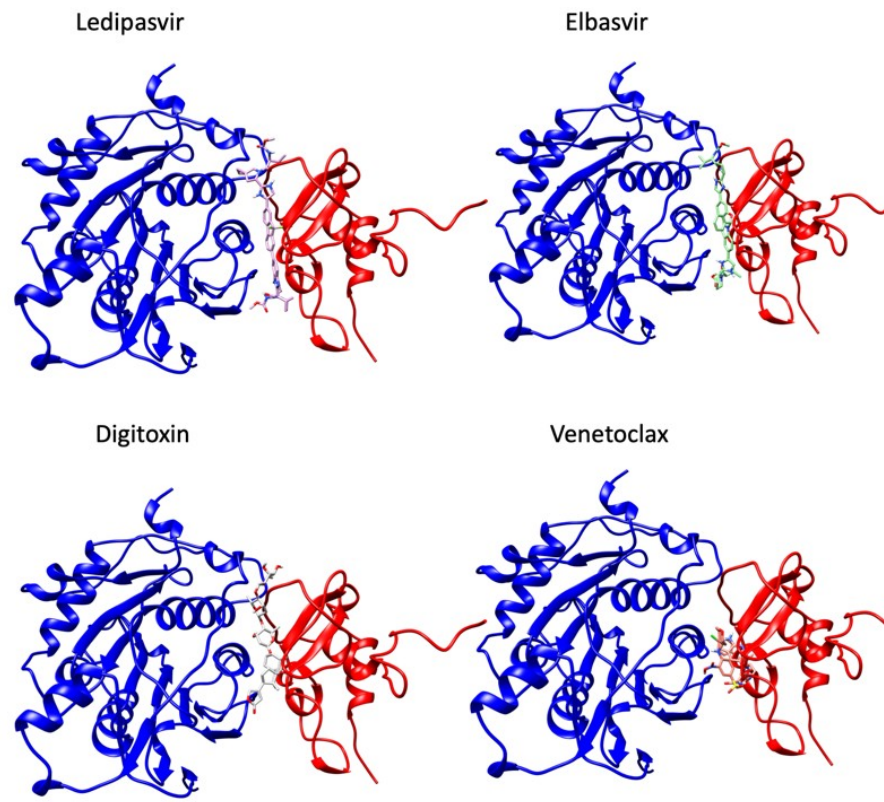

Figure 6: Visualization of Nsp16-Nsp10 complex with a docked drug molecule. Note that in docking calculations, only Nsp16 is used. Blue color shows Nsp16, and Red color shows Nsp10 proteins.

Current use of the drugs predicted in this work was searched in the literature. Dexamethasone is currently being used for the treatment of hospitalized patients. In randomized clinical trials (RCT) Dexamethasone significantly lowered the mortality rate (23). Ledipasvir/sofosbuvir combination did not exhibit significant efficacy in a very small RCT, yet RCTs with larger sample sizes are needed to assess the efficacy of Ledipasvir (24). Venetoclax is being tested on Covid-19 fit patients with Chronic lymphocytic leukemia (25). There is no RCT study on Digitoxin but there is an experimental evidence on impeding the cytokine storm which eventually could lead to the death of Covid-19 patients (26). Simeprevir has not been tested in an RCT but in combination with remdesivir it can suppress the replication of SARS-CoV-2 in vitro (27). No clinical trial has been performed on Elbasvir, Irinotecan, Acetyldigitoxin, Dactinomycin and Lumacaftor.

\section{CONCLUSION}

The interaction between Nsp16 and Nsp10 is essential for SARS-CoV-2 to escape from the host immune system. In this work, potential drug molecules which could prevent the formation of the Nsp16-Nsp10 complex were predicted using docking calculations. Drugs Ledipasvir Elbasvir, Venetoclax, Digitoxin, Irinotecan, Dexamethasone, Acetyldigitoxin, Dactinomycin, Lumacaftor, and Simeprevir were ranked in the top-10 by their docking scores. Among other amino acids of Nsp16, Gln6885, Ser6903, Val6842, Ala6881, Phe7043, Leu7042, Asp6904, and Arg6884 were predicted to be essential amino acids for the interaction with drug molecules. Drug molecules predicted in this study will open new possibilities for the treatment of SARS-CoV-2 infected patients.

\section{REFERENCES}

1. WHO. Coronavirus disease (COVID-19) pandemic [Internet]. World Health Organization; 2021 [cited 2021 Aug 5]. $\leq$ URL>

2. Madhi SA, Baillie V, Cutland CL, Voysey M, Koen AL, Fairlie L, et al. Efficacy of the ChAdOx1 nCoV-19 Covid-19 Vaccine against the B.1.351 Variant. N Engl J Med. 2021 May 20;384(20):1885-98. $\leq \mathrm{DOI}>$.

3. Durdagi S. Virtual drug repurposing study against SARS-CoV-2 TMPRSS2 target. Turkish Journal of Biology. 2020 Jun $21 ; 44($ SI-1):185-91. $\leq$ URL $>$.

4. Gordon DE, Jang GM, Bouhaddou M, Xu J, Obernier K, White $\mathrm{KM}$, et al. A SARS-CoV-2 protein interaction map reveals targets for drug repurposing. Nature. $2020 \mathrm{Jul}$ 16;583(7816):459-68. <DOI>.

5. Idris MO, Yekeen AA, Alakanse OS, Durojaye OA. Computer-aided screening for potential TMPRSS2 inhibitors: a combination of pharmacophore modeling, molecular docking and molecular dynamics simulation approaches. Journal of Biomolecular Structure and Dynamics. 2020 Jul 16;1-19. <DOI>.

6. Choudhary S, Malik YS, Tomar S. Identification of SARSCoV-2 Cell Entry Inhibitors by Drug Repurposing Using in silico Structure-Based Virtual Screening Approach. Front Immunol. 2020 Jul 10;11:1664. <DOI $>$.

7. Baby K, Maity S, Mehta CH, Suresh A, Nayak UY, Nayak Y. Targeting SARS-CoV-2 Main Protease: A Computational Drug Repurposing Study. Archives of Medical Research. 2021 Jan;52(1):38-47. $\leq$ DOI $>$. 
8. Lin $\mathrm{S}$, Chen $\mathrm{H}, \mathrm{Ye} F$, Chen $\mathrm{Z}$, Yang $\mathrm{F}$, Zheng $\mathrm{Y}$, et al. Crystal structure of SARS-CoV-2 nsp10/nsp16 2'-Omethylase and its implication on antiviral drug design. Sig

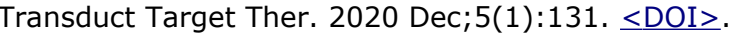

9. Vithani N, Ward MD, Zimmerman MI, Novak B, Borowsky JH, Singh S, et al. SARS-CoV-2 Nsp16 activation mechanism and a cryptic pocket with pan-coronavirus antiviral potential. Biophysical Journal. 2021 Jul;120(14):2880-9. <DOI >.

10. El Khatabi K, Aanouz İ, El-MerniSsi R, Khaldan A, Ajana $M A$, Bouachrine $M$, et al. Designing of Novel Potential Inhibitors of a-amylase by 3D-QSAR Modeling and Molecular Docking Studies. Journal of the Turkish Chemical Society Section A: Chemistry. 2020 May 10;469-78. $<$ DOI $>$.

11. Sulaiman M, Hassan $Y$, TaskiN Tok T, Noundou XS. SYNTHESIS, ANTIBACTERIAL ACTIVITY AND DOCKING STUDIES OF BENZYL ALCOHOL DERIVATIVES. Journal of the Turkish Chemical Society Section A: Chemistry. 2020 May $11 ; 481-8$. $\leq$ DOI $>$.

12. Kiliç Kurt Z. Synthesis of Novel Oxadiazole Derivatives, Molecular Properties Prediction and Molecular Docking Studies. Journal of the Turkish Chemical Society Section A: Chemistry. 2020 Oct 30;7(3):753-74. <DOI>.

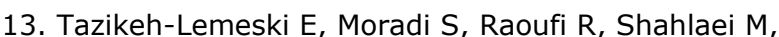
Janlou MAM, Zolghadri S. Targeting SARS-COV-2 nonstructural protein 16: a virtual drug repurposing study. Journal of Biomolecular Structure and Dynamics. $2021 \mathrm{Sep}$ 2;39(13):4633-46. <DOI >.

14. Jiang $Y$, Liu L, Manning M, Bonahoom M, Lotvola A, Yang Z-Q. Repurposing Therapeutics to Identify Novel Inhibitors Targeting 2'-O-Ribose Methyltransferase Nsp16 of SARS-CoV-2 [Internet]. 2020 May [cited 2021 Aug 8]. $\leq$ URL > . Note: This content is a preprint and has not been peer-reviewed.

15. Vijayan V, Pant P, Vikram N, Kaur P, Singh TP, Sharma $S$, et al. Identification of promising drug candidates against NSP16 of SARS-CoV-2 through computational drug repurposing study. Journal of Biomolecular Structure and Dynamics. 2020 Aug 3;1-15. <DOI $>$.

16. Olsson M, Søndergard C, Rostkowski M, Jensen J. PROPKA3: Consistent Treatment of Internal and Surface Residues in Empirical pKa predictions. J Chem Theo Comput. $2011 ;(2): 525-37$.

17. Søndergaard CR, Olsson MHM, Rostkowski M, Jensen $\mathrm{JH}$. Improved Treatment of Ligands and Coupling Effects in
Empirical Calculation and Rationalization of $\mathrm{p} \mathrm{K}$ a Values. J Chem Theory Comput. 2011 Jul 12;7(7):2284-95. <DOI>.

18. Morris GM, Huey R, Lindstrom W, Sanner MF, Belew RK, Goodsell DS, et al. AutoDock4 and AutoDockTools4: Automated docking with selective receptor flexibility. J Comput Chem. 2009 Dec;30(16):2785-91. <DOI $>$.

19. Trott O, Olson AJ. AutoDock Vina: Improving the speed and accuracy of docking with a new scoring function, efficient optimization, and multithreading. J Comput Chem. 2009;455-61. <DOI>.

20. Wishart DS, Feunang YD, Guo AC, Lo EJ, Marcu A, Grant JR, et al. DrugBank 5.0: a major update to the DrugBank database for 2018. Nucleic Acids Research. 2018 Jan 4;46(D1):D1074-82. <DOI>.

21. O'Boyle NM, Banck M, James CA, Morley C, Vandermeersch T, Hutchison GR. Open Babel: An open chemical toolbox. J Cheminform. $2011 \mathrm{Dec} ; 3(1): 33$. $<$ DOI $>$.

22. Maestro. Schrödinger Release 2016-4 [Internet]. Schrödinger, LLC; 2016 [cited 2021 Aug 5]. <URL>.

23. RECOVERY Collaborative Group, Horby $P$, Lim WS, Emberson JR, Mafham M, Bell JL, et al. Dexamethasone in Hospitalized Patients with Covid-19. N Engl J Med. 2021 Feb 25;384(8):693-704. <DOI $>$.

24. Khalili H, Nourian A, Ahmadinejad Z, Emadi Kouchak $\mathrm{H}$, Jafari S, Dehghan Manshadi SA, et al. Efficacy and safety of sofosbuvir/ ledipasvir in treatment of patients with COVID-19; A randomized clinical trial. Acta Biomed. 2020 Nov 10;91(4):e2020102. <DOI>.

25. Fürstenau $M$, Langerbeins $P$, De Silva $N$, Fink $A M$, Robrecht S, von Tresckow J, et al. COVID-19 among fit patients with CLL treated with venetoclax-based combinations. Leukemia. 2020 Aug;34(8):2225-9. $\leq \mathrm{DOI}>$.

26. Pollard BS, BLANCOI JC, Pollard JR. Classical Drug Digitoxin Inhibits Influenza Cytokine Storm, With Implications for Covid-19 Therapy. In Vivo. 2020;34(6):3723-30. <DOI .

27. Lo HS, Hui KPY, Lai H-M, He X, Khan KS, Kaur S, et al. Simeprevir Potently Suppresses SARS-CoV-2 Replication and Synergizes with Remdesivir. ACS Cent Sci. 2021 May 26;7(5):792-802. $\leq$ DOI $>$. 